\title{
Step-Response Analysis of Chemotaxis in Caenorhabditis elegans
}

\author{
Adam C. Miller, ${ }^{\star}$ Tod R. Thiele, ${ }^{\star}$ Serge Faumont, ${ }^{\star}$ Marin L. Moravec, and Shawn R. Lockery \\ Institute of Neuroscience, University of Oregon, Eugene, Oregon 97403
}

The sensorimotor transformation underlying Caenorhabditis elegans chemotaxis has been difficult to measure directly under normal assay conditions. Thus, key features of this transformation remain obscure, such as its time course and dependence on stimulus amplitude. Here, we present a comprehensive characterization of the transformation as obtained by inducing stepwise temporal changes in attractant concentration within the substrate as the worm crawls across it. We found that the step response is complex, with multiple phases and a nonlinear dependence on the sign and amplitude of the stimulus. Nevertheless, the step response could be reduced to a simple kinetic model that predicted the results of chemotaxis assays. Analysis of the model showed that chemotaxis results from the combined effects of approach and avoidance responses to concentration increases and decreases, respectively. Surprisingly, ablation of the ASE chemosensory neurons, known to be necessary for chemotaxis in chemical gradient assays, eliminated avoidance responses but left approach responses intact. These results indicate that the transformation can be dissected into components to which identified neurons can be assigned.

Key words: C. elegans; nematode; chemotaxis; sensorimotor integration; chemosensory neurons; stochastic models

\section{Introduction}

The chemotaxis behavior of the nematode Caenorhabditis elegans raises, in simplified form, the key question of how the nervous system transforms sensory input into motor output to regulate a goal-directed response. Odorants and tastants are encoded by an array of just 11 pairs of identified chemosensory neurons (Ward et al., 1975; Ware et al., 1975). Motor output is also simple, comprising periods of forward movement punctuated approximately twice per minute by bouts of turning (Rutherford and Croll, 1979). Two main kinds of turns are recognized in C. elegans: "reversals," in which the animal first moves backward for several seconds and then forward again in a new direction, and "omega turns," in which the animal bends head to tail while moving forward, momentarily forming a shape like the Greek letter (Croll, 1975). Reversals and omega turns occur in bursts that have been termed pirouettes (Pierce-Shimomura et al., 1999). Our goal is to understand at the neuronal level how chemosensory input regulates turning to produce chemotaxis.

A key step in the neuronal analysis of a behavior is to identify its sensorimotor transformation (i.e., the function that relates the amplitude and waveform of the stimulus to the amplitude and

Received Dec. 15, 2004; revised Feb. 3, 2005; accepted Feb. 16, 2005.

This work was supported by National Institute of Mental Health Grant MH051383. Nematode strains were provided by the Caenorhabditis Genetics Center, which is funded by the National Institutes of Health-National Center for Research Resources. We thank William Roberts for help in implementing the Q-matrix method.

${ }^{*}$ A.C.M., T.R.T., and S.F. contributed equally to this work.

Correspondence should be addressed to Shawn R. Lockery, Institute of Neuroscience, University of Oregon, 1254 University of Oregon, Eugene, OR 97403-1254. E-mail: shawn@lox.uoregon.edu.

M. L. Moravec's present address: Department of Ecology and Evolutionary Biology, University of California, Irvine, Irvine, CA 92697.

DOI:10.1523/JNEUROSCI.5133-04.2005

Copyright $\odot 2005$ Society for Neuroscience $\quad$ 0270-6474/05/253369-10\$15.00/0 waveform of the response). Chemotaxis in C. elegans is studied in two main types of gradients: steep gradients with step-like concentration borders (Jansen et al., 2002) and shallow gradients formed by radial diffusion from a point bolus of attractant (Ward, 1973). Previous studies in which worms were tracked during chemotaxis in a shallow gradient showed that pirouette probability is modulated by the time rate of change of chemical concentration $(d C / d t)$, such that when $d C / d t<0$, pirouette probability is increased, whereas when $d C / d t>0$, pirouette probability is decreased (Pierce-Shimomura et al., 1999). Thus, excursions down the gradient are truncated, whereas excursions up the gradient are extended, resulting in net movement toward the gradient peak. Tracking experiments, however, are generally insufficient to define a sensorimotor transformation, because stimulus and behavior change continuously as a worm moves within a spatial gradient. The most direct way to disentangle stimulus and behavior would be to observe the response to a spatially uniform concentration step or impulse (Dusenbery, 1980; Block et al., 1982; Segall et al., 1986). Such stimuli have the additional advantage that they can be used to predict the response to an arbitrary input waveform (provided the sensorimotor transformation is linear). In C. elegans, however, neither steps nor impulses have been achieved under normal assay conditions in which the worm is crawling on an agar surface.

Here, we present a comprehensive characterization of the chemotaxis sensorimotor transformation in terms of the behavioral response to concentration steps experienced in steep gradients. This was accomplished using a novel concentration-clamp apparatus designed to produce stepwise temporal changes in attractant concentration within the substrate beneath the worm. We found that the step responses likely to underlie C. elegans chemotaxis in steep gradients are complex, with multiple phases and a 
nonlinear dependence on the sign and amplitude of the stimulus. Despite these complexities, the sensorimotor transformation could be described by a simple three-state kinetic model that accurately predicts the behavior of populations of worms in chemotaxis assays. Our analysis suggests that chemotaxis is the joint result of avoidance responses comprising turns triggered by concentration decreases and approach responses comprising runs triggered by concentration increases. Surprisingly, ablation of key chemosensory neurons eliminated avoidance responses but left approach responses intact. This result indicates that there are separate neuronal pathways for approach and avoidance behaviors within the chemotaxis network and that individual neurons can be assigned to these pathways.

\section{Materials and Methods}

Animals. The strains used in this study were wild-type (WT) C. elegans Bristol (N2) and che-1 (p679). Nematodes were grown in mixed-stage cultures at room temperature $\left(22-25^{\circ} \mathrm{C}\right)$ on $1.7 \%$ agar-filled plates containing nematode growth medium seeded with Escherichia coli strain OP50 (Brenner, 1974). All experiments were performed at room temperature $\left(22-25^{\circ} \mathrm{C}\right)$.

Solutions. Saline solutions for step-response assays contained only $\mathrm{NaCl}(0,20,40,50,60,80,100$, and $150 \mathrm{~mm})$ and sorbitol to a total osmolarity of $350 \mathrm{mOsm}$. The $\mathrm{pH}$ was set to 6.2 using $\mathrm{NaOH}$; buffer was omitted because it reduced the level of chemotaxis in the quadrant assays. For quadrant chemotaxis assays, agarose $(1.7 \%)$ was added to the stepresponse solutions.

Quadrant assays. Gradients with abrupt concentration borders were constructed using Petri plates divided into quadrants (X-plates; diameter, $9 \mathrm{~cm}$; Fisher Scientific, Houston, TX), such that opposing quadrants contained either agarose with $50 \mathrm{~mm} \mathrm{NaCl}$ or one of eight different test concentrations of $\mathrm{NaCl}$ (Wicks et al., 2000). To prevent behavioral interference from the plastic barriers in the plate, quadrants were slightly overfilled (to a positive meniscus) with molten agarose. Then, after the agarose solidified, we coated the surface of the plate with a thin $(1 \mathrm{~mm})$ layer of molten agarose (5 $\mathrm{ml}$ of $1.7 \%$ agarose in distilled water). To allow for diffusion of $\mathrm{NaCl}$ from the quadrants into the thin agarose layer, 25 min elapsed before the assay began. Animals grown in mixed-stage cultures were rinsed twice in distilled water to remove bacteria and to segregate adults from larval animals. Between 50 and 100 young adults were transferred in a distilled water-filled capillary to the center of the quadrant plate. Excess water was removed by suction. Animals were allowed to move for $10 \mathrm{~min}$, at which point the number of worms in each quadrant was recorded. The chemotaxis index $I$ was computed as $I=\left(N_{50}-\right.$ $\left.N_{\text {Test }}\right) /\left(N_{50}+N_{\text {Test }}\right)$, where $N_{50}$ is the number of worms in the $50 \mathrm{~mm}$ quadrants and $N_{\text {Test }}$ is the number of worms in the quadrants containing the test concentration.

Step-response apparatus. The apparatus for delivering rapid chemical steps is depicted in Figure $2 \mathrm{~A}$. The behavioral substrate consisted of an agarose-coated polycarbonate membrane (10.0 $\mu \mathrm{M}$; Isopore; Millipore, Billerica, MA). The agarose coating was formed by casting a molten $0.4 \%$ agarose solution (low electro-endo-osmosis electrophoresis grade; Fisher Scientific) to a depth of $4 \mathrm{~mm}$ in a circular dam. After the agarose solidified, the membrane was dried in a $70^{\circ} \mathrm{C}$ oven for $1 \mathrm{~h}$. The membrane was glued to a glass frame using cyanoacrylate adhesive and suspended above the showerheads, such that the underside of the membrane was $2 \mathrm{~mm}$ from the top of the showerheads. Concentration changes in time were produced by moving the showerheads from side to side; showerheads were mounted on a smooth, linear translator to reduce vibrations when sliding. Solutions were pumped through the showerheads at a rate of 1 $\mathrm{ml} / \mathrm{min}$ using a peristaltic pump. Fluid flowed radially, from the center of each showerhead to a circular gutter in the perimeter. The rate of solution exchange was ascertained in separate experiments via the time course of the electrical potential difference between recording sites above and below the membrane as the showerhead assembly was moved to simulate a step-response experiment. The upper site was monitored by a $3 \mathrm{M} \mathrm{KCl}$ electrode; the lower site was monitored by a silver chloride electrode inserted into the port at the center of the high $\mathrm{NaCl}$ showerhead. The half-time of the concentration change was $1.3-2.6 \mathrm{~s}$.

Definitions of behaviors. The behavioral criteria by which we scored behavioral transitions were designed to both accurately reflect the nature of the transitions and make it easier for a human observer to score them objectively and reliably. Accordingly, behavior was analyzed in terms of three main behavioral states: forward, reversal, and omega turn; these three states accounted for $98 \%$ of total observation time in our experiments. A fourth state, called "other," was used for the rare instances in which behavior fell into none of the three main categories. The forward state was defined as anterior translation of the body associated with a head-to-tail body wave. The reversal state was defined as caudal translation of the body associated with a tail-to-head body wave. The omegaturn state was defined as head-to-body contact with the head moving forward. Note that our classification scheme omits deep bends, which resemble omega turns, except that there is no contact between head and tail. This omission was unavoidable, because deep bends are graded in amplitude between the bends of normal swimming movements and omega turns; thus, deep bends are difficult to discern objectively. In a three-state classification system, there are six possible state transitions. We used the following objective criteria to define the instant at which a given transition occurred: forward-reversal (FR) (first backward movement of the tail), reversal-forward (RF) (first forward movement of the head), forwardomega ( $F \Omega$ ) (the instant head-body contact was made), omega-forward $(\Omega \mathrm{F})$ (the instant head-body contact was broken), omega-reversal $(\Omega \mathrm{R})$ (first backward movement of the tail), and reversal-omega (R $\Omega$ ) (the instant head-body contact was made).

Step-response assay. Young adults isolated the day before as L4 larvae were picked to an unseeded nematode growth medium plate to remove adherent bacteria. Animals were then transferred in a saline-filled pipette to the behavioral substrate. The transfer solution corresponded to the first solution that the animal was to encounter in the assay. Excess transfer solution was removed with a pointed piece of filter paper. To eliminate handling artifacts, such as the mechanically induced suppression of reversals (Zhao et al., 2003), we waited 2 min before recording behavior. The behavior of individual worms was scored visually by a human observer who pressed computer keys to note the initial behavioral state and the time and type of each state transition thereafter. Visual, as opposed to automated, scoring was necessary because the concentration clamp imparts a visually complex and moving background (supplemental movie 1, available at www.jneurosci.org as supplemental material), making it unusually difficult to segment the image automatically.

We assessed the reliability of our scoring method in two ways: between observers and within observers. For between-observer comparisons, four different observers scored the same videotape of two worms crawling for 5 min each under normal experimental conditions. Between-observer reliability was quantified by computing the SD of the four probability scores for each $10 \mathrm{~s}$ time bin in the video. The SD of forward probability scores, the main dependent variable in our study, was $0.0-0.076$ probability units, with an average of 0.028 probability units. Because average forward probability is 0.8 , the average SD represents an error of $3.5 \%$. For within-observer comparisons, a single observer scored the videotape four times. Here, the SD for forward probability was $0.0-0.055$ probability units, with an average of 0.020 probability units, which corresponds to an error of $2.5 \%$. Thus, our scoring method was reliable both between and within observers.

Visual observation could, in principle, have introduced two main kinds of error. First, between-observer variability (there were two observers in this study) could have increased the variance about the mean probabilities, such as those plotted in Figure 3. The effect of this type of error would be to make it harder to detect behavioral responses to concentration steps; this type of error is therefore conservative in the context of our study. Second, visual observation could have introduced a systematic bias caused by observer reaction time. Plotting the distribution of dwell times in each state, we noted that there were fewer dwell times $\leq 2 \mathrm{~s}$ than expected based on the an exponential fit to the data; thus, we estimate that the scorer's reaction time was $\sim 2 \mathrm{~s}$. Transition events missed because of reaction-time errors could have caused us to underestimate the transition rate constants $k_{i j}=f_{i j} / p_{i}$. This is because $f_{i j}$ is more 
sensitive to missing brief events than is $p_{i}$. However, reaction-time error was unlikely to have affected our estimates of state probabilities, because it adds equally to the time of behavioral onset and offset.

Statistics. In step-response experiments, behavioral-state probabilities ( $p$ ) were transformed as $2 \arcsin (\sqrt{p})$ to compensate for compression of the variance at the extremes of the probability scale (Winer et al., 1991); this transformation is conservative because it increases the variance at the extremes. Statistical significance was assessed by a two-factor repeatedmeasures ANOVA applied to each of the two time windows indicated by the horizontal lines above the probability traces in Figures 3 and 6 . The length of the two windows in Figure 3 was fixed a priori from the durations of the facilitation or suppression of reversals in response to stepwise temporal changes in $\mathrm{NaCl}$ concentration in swimming worms (Dusenbery, 1980). The length of the time windows in Figure 6 was determined by the durations of effects in Figure 3. In addition, we made planned pairwise comparisons ( $t$ tests) on mean behavioral-state probabilities at each time point in the analysis window. The significance level at each time point was adjusted using Bonferroni's correction to compensate for the fact that multiple comparisons increase the false positive rate. Because Bonferroni's correction is conservative, significant effects in uncorrected $t$ tests are also shown as an upper bound on the number of significant pairwise comparisons.

Laser ablations. Cells were killed as described previously (Bargmann and Avery, 1995). L1 animals (N2) were mounted on 5\% agarose pads containing 5-7 mm of the immobilizing agent $\mathrm{NaN}_{3}$. ASE neurons were identified by position. Animals were remounted $1-3 \mathrm{~h}$ after surgery to confirm ablation and to look for possible collateral damage; damaged animals were discarded. Sham-operated animals were treated in the same manner, except that the laser was not fired. Behavior was tested $3 \mathrm{~d}$ after ablation; the experimenter was blind to animal treatment.

Kinetic model of chemotaxis. Our kinetic model assumes that transitions out of a state occur at a constant rate, regardless of how long the system has been in the state. Although this is mostly true (see Fig. 4A), additional statistical analysis of behavioral transitions revealed two higher-order dependencies in the state transitions that are mentioned here for completeness. (1) The likelihoods of the two types of transitions out of the reversal and omega states depended on how long the system was in the state (data not shown), consistent with previous observations (Zhao et al., 2003). Presumably, the changes in these rates compensate each other, yielding the results shown in Figure $4 \mathrm{~A}$. Importantly, however, no such dependencies were found for transitions from the forward state, which is the state most often occupied. (2) Using a test for sequential dependencies (Haccou and Meelis, 1992), we found that the likelihoods of two of the six possible transitions were sensitive to which state preceded the transition; the affected transitions were forward-reversal $\left[\chi^{2}(2)=32.78 ; p<0.001\right]$ and forward-omega $\left[\chi^{2}(2)=53.63 ; p<\right.$ $0.001]$. Although the model omitted both types of higher-order dependency, it nevertheless reproduced the main features of the quadrant assays, and it did so with no free parameters. Thus, the higher-order dependencies are not essential for understanding the relationship between step responses and chemotaxis behavior.

Simulated quadrant assays. The model worm's initial location and orientation were randomized within a 4 -mm-diameter circle at the center of the virtual assay plate (diameter, $9 \mathrm{~cm}$ ). Time-dependent quantities were updated at $1 \mathrm{~s}$ intervals. A matrix $K$ of current transition rates was maintained by convolving the time courses of changes in rate constants $\Delta k_{i j}(t)$ with border crossings between regions of different concentration in the virtual assay plate. At the beginning of each time interval $\Delta t$, a behavioral state was selected randomly according to the current probability of each state. Current probability was evaluated by computing the relaxation of state probabilities toward their equilibrium probabilities from the initial condition specified by the state of the system at the beginning of the previous interval. This computation was done according to the Q-matrix method (Colquhoun and Hawkes, 1995), using the following equation: $\boldsymbol{p}(t+\Delta t)=\boldsymbol{p}(t)$ $\exp (\boldsymbol{Q} \Delta t)$, where $\boldsymbol{p}(t+\Delta t)$ is the vector of current state probabilities, $\boldsymbol{p}(t)$ is a vector of $0 \mathrm{~s}$ and $1 \mathrm{~s}$, representing the state of the system at the beginning of previous interval, and $\boldsymbol{Q}$ is the matrix $\boldsymbol{K}$ with the diagonal terms $q_{i i}$ set to the opposite of the exit rate for state $i$. If no change in state occurred, the worm continued to move with the original speed and orientation. However, if there was a change in state, the worm's orientation was updated by the angle $\Delta \theta$ chosen in one of two ways. (1) For the transitions $\mathrm{F} \Omega, \mathrm{RF}$, and $\mathrm{R} \Omega, \Delta \theta$ was selected according to the empirical distributions in Figure $4 C$. These distributions were obtained by measuring turning angle by hand in video recordings of worms crawling on foodless agarose plates in the absence of a concentration gradient. The data were plotted as if each worm were crawling on its right side and making omega turns to its ventral side. The number of turns observed were as follows: $F \Omega, 156 ; \mathrm{RF}, 35$; and $\mathrm{R} \Omega$, 104. (2) For the other three transitions, $\Delta \theta$ was a fixed value that represented the effect of the transition in idealized form: $\mathrm{FR}, \Delta \theta=180^{\circ}$, because reversals are comparatively straight (Zhao et al., 2003); $\Omega \mathrm{F}, \Delta \theta=0^{\circ}$, because the worm is already moving forward during an omega turn by definition; $\Omega \mathrm{R}, \Delta \theta=180^{\circ}$, because, in the model, the worm exits the omega state moving forward, so $\Omega \mathrm{R}$ is equivalent to the three-state sequence $\Omega$ FR. Speed in the forward and reversal states was $0.2 \mathrm{~mm} / \mathrm{s}$; in the omega state, it was $0 \mathrm{~mm} / \mathrm{s}$. Each model worm was "run" for $10 \mathrm{~min}$ of virtual time in accordance with run durations in the real assays; there were 2000 model worms per assay.

\section{Results}

\section{Quadrant assays}

To determine the range of concentration steps likely to produce detectable changes in turning behavior, we measured chemotaxis ability as a function of concentration differences in steep spatial gradients with abrupt concentration borders. This experiment is based on the fact that a worm crossing an abrupt concentration border experiences a stepwise concentration change in time. Gradients with abrupt borders were formed in agar-filled Petri plates divided into quadrants (Wicks et al., 2000). Diametrically opposed quadrants contained either $50 \mathrm{~mm} \mathrm{NaCl}$, the home concentration at which the worms were cultivated, or one of eight different test concentrations. Test concentrations were chosen to produce concentration changes of $\pm 10, \pm 30, \pm 50$, and \pm 100 $\mathrm{mm}$ as a worm crossed the border between quadrants. Chemotaxis was quantified in terms of an index $I$ that reflected the worm's preference for the home concentration $(I>0)$ or the test concentration $(I<0)$. Chemotaxis index was strongly influenced by the test concentration (Fig. 1) $\left(F_{(7,120)}=20.00 ; p<0.001\right)$. When the test concentration was less than the home concentration, the worms preferred the home concentration, whereas when the test concentration was greater than the home concentration, the worms preferred the test concentration. Most of the data lay along a negatively sloping line. We noticed, however, that worms were not as strongly attracted to $150 \mathrm{~mm} \mathrm{NaCl}$, as this trend predicts; this observation is addressed further below.

We draw two conclusions from this experiment. First, concentration steps in the range of \pm 10 to $\pm 100 \mathrm{~mm}$ are behaviorally relevant for chemotaxis at the population level and thus are likely to produce detectable changes in turning behavior in a step-response experiment. Second, the worm's overall goal in chemotaxis to $\mathrm{NaCl}$ in this concentration range is to maximize concentration rather than to return to the home concentration. In contrast, the thermotaxis response in $C$. elegans returns the animal to its home temperature (Hedgecock and Russell, 1975). Thus, C. elegans chemotaxis and thermotaxis differ in this fundamental respect.

\section{Step-response assays}

To study the step responses, worms were placed individually on a thin porous membrane supported over a yoked pair of miniature inverted showerheads (Fig. $2 A$ ). Each showerhead emitted a saline solution with a different salt concentration. The half-time for solution exchange at the top of the membrane, monitored electrochemically as described in Materials and Methods, was 1.3-2.6 s. Stepwise temporal changes in salt concentration were delivered by sliding the showerhead assembly relative to the worm. We 
measured the effect on behavior of concentration steps of \pm 10 , \pm 50 , and \pm 100 from a variety of baseline concentrations. Step amplitudes and baseline concentrations were selected to simulate the concentration changes experienced by worms in quadrant assays a-f, as shown on Figure 1. For example, assay a was simulated by a $+50 \mathrm{~mm}$ step from a baseline of $0 \mathrm{~mm}$ and a $-50 \mathrm{~mm}$ step from a baseline of $50 \mathrm{~mm}$.

A typical step-response experiment is illustrated in Figure $2 B$. Behavior was recorded in the form of an ethogram by noting the times at which transitions occurred among four states: forward, reversal, omega turn, and other, as defined in Materials and Methods. Here, we displayed in raster format the ethograms of 30 worms subjected to a $+50 \mathrm{~mm}$ concentration step from a baseline concentration of $50 \mathrm{~mm}$. Each worm's ethogram was converted into four separate functions of probability versus time, one for each behavioral state, and ensemble averages were computed across worms, yielding the traces shown in Figure 2C. In this experiment, the probability of the forward state increased, whereas the probabilities of the reversal and omega-turn states decreased, corresponding to a decline in pirouette probability. This pattern of reciprocal regulation of forward and turn states was a salient feature of the response to other concentration steps, as can be seen within rows in Table 1 . In no-step control experiments in which both showerheads emitted the same salt concentration $(50 \mathrm{~mm})$, probability time courses for all behaviors were flat, indicating that the step responses were not influenced by the mechanical correlates of the concentration change (Fig. 2C).

\section{Step responses}

We observed statistically significant changes in behavioral-state probabilities for at least one behavioral state in response to each combination of step amplitude and baseline concentration (Fig. 3, Table 1). Significance was assessed by comparing the step and no-step conditions. The initial effect of upsteps (Fig. 3, left column) $(t=0$ to $\approx 50 \mathrm{~s}$ ) was an increase in forward probability with a concomitant decrease in reversal and/or omega probabilities; we use the term run to refer to this configuration of responses. Conversely, the initial effect of downsteps (Fig. 3, right column) $(t=0$ to $\approx 20 \mathrm{~s}$ ) was a decrease in forward probability with a concomitant increase in reversal and/or omega probabilities; we use the term "turn" to refer to this configuration of responses.

As the amplitude of concentration steps increased, step-response waveforms changed from monophasic to biphasic, with distinct early and late components. In particular, large downsteps $(\Delta C=-50$ or $-100 \mathrm{~mm}$ ) produced the sequence "turnand-run" (Fig. $3 F, H, J$ ), whereas large upsteps $(\Delta C=100 \mathrm{~mm})$ produced the sequence "run-and-turn" (Fig. 3I). Two lines of evidence argue that the lateresponse component was not attributable to an effect of concentration on steadystate behavior. First, in three of four cases in which the late component was observed, the forward probability returned to the prestimulus baseline level at the end of the

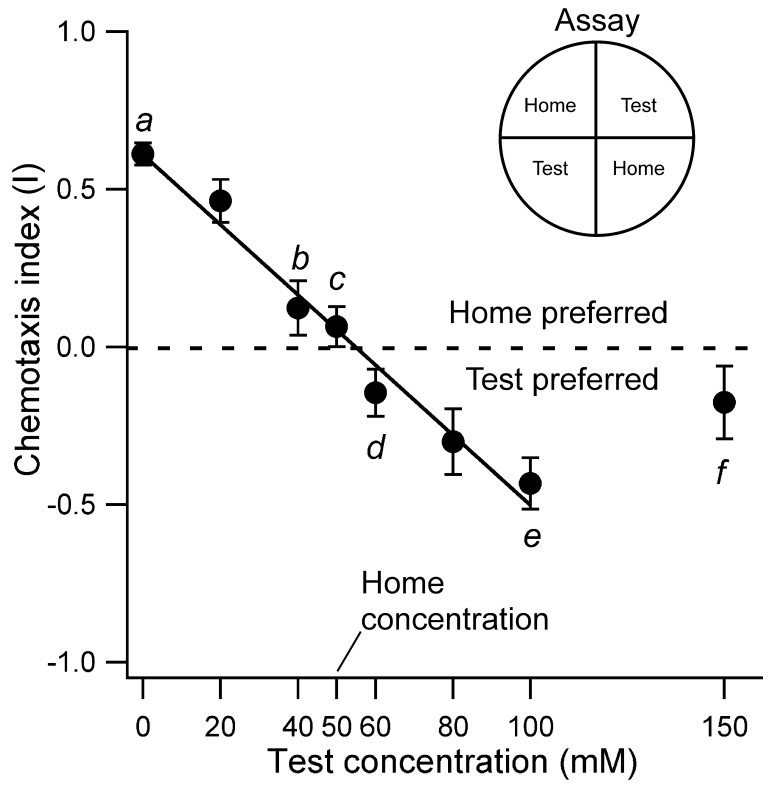

Figure 1. Chemotaxis index versus test concentration in the quadrant assay. Approximately 100 worms were placed at the center of the plate (inset) and allowed to move for $10 \mathrm{~min}$. The index was computed as described in Materials and Methods, such that $I>0$ reflects a preference for the home concentration of $\mathrm{NaCl}(50 \mathrm{~mm})$, whereas $/<0$ reflects a preference for the test concentration. Each point represents the mean value of at least 16 assay plates. The line is a linear fit to the data for test concentrations from 0 to $100 \mathrm{~mm}$. Letters a-f correspond to rows in Table 1. Overall, worms moved so as to maximize the ambient $\mathrm{NaCl}$ concentration. This effect was weaker than expected at a test concentration of $150 \mathrm{~mm}$. Inset, Diagram of the quadrant assay.
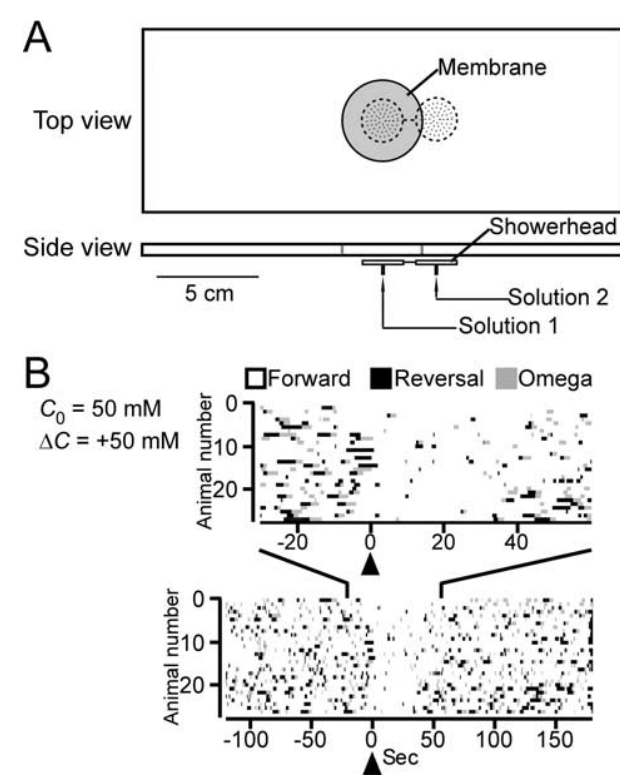
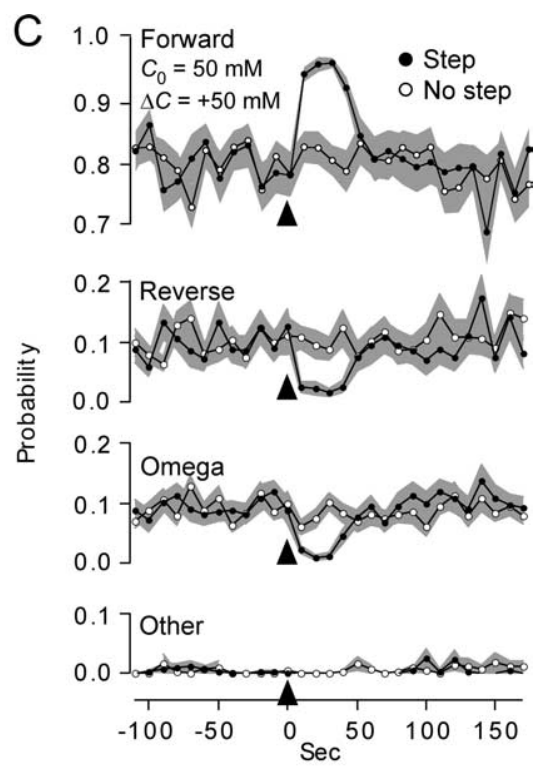

Figure 2. The step-response assay. $\boldsymbol{A}$, Apparatus. A thin $(8 \mu \mathrm{m})$ porous membrane was suspended over a pair of inverted showerheads. Each showerhead emitted a saline solution with a different $\mathrm{NaCl}$ concentration. Stepwise concentration changes were induced within the membrane by sliding the showerhead assembly sideways. $\boldsymbol{B}$, Ethograms. Each row in the raster plot shows the behavioral record for a single worm before and after a concentration step $(\Delta C)$ of $+50 \mathrm{~mm}$ from a baseline concentra$\operatorname{tion}\left(C_{0}\right)$ of $50 \mathrm{~mm}$. The top panel is an expanded view of the bottom panel as indicated by the lines between panels. White regions indicate the forward state, black regions indicate the reversal state, and gray regions indicate the omega state. In both panels, the concentration step occurred at 0 s. C, Behavioral-state probabilities as a function of time for the experiment shown in $\boldsymbol{B}$. Individual worm ethograms were converted in four separate functions of state probability versus time by computing the fraction of time spent in each state in $10 \mathrm{~s}$ bins. Probability data were summarized by computing ensemble averages (across worms) of the probability of each behavioral state as a function of time relative to the concentration step. Arrowheads indicate the time of the concentration step. SEM is shown by the gray bands. 

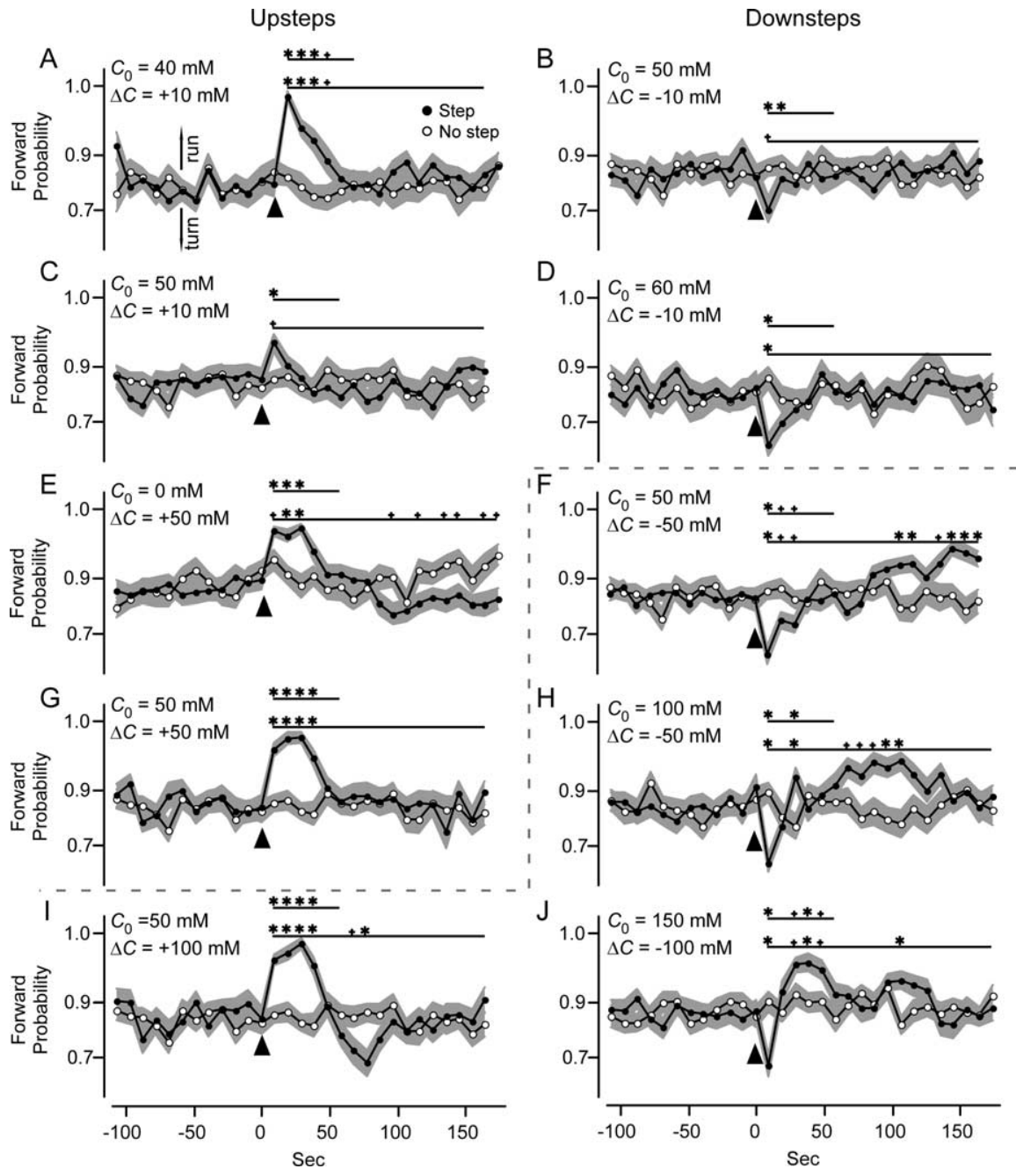

Figure 3. Step-response analysis of the chemotaxis sensorimotor transformation. Each panel shows the time course of average forward-state probability as a function of time for the baseline concentration of $\mathrm{NaCl}\left(C_{0}\right)$ and concentration step $(\Delta C)$ shown in the top left corner. $\boldsymbol{A}$, Symbols are given in the key. Arrowheads indicate the time of the concentration step. In control experiments (no step), both showerheads emitted the baseline concentration. Excursions in forward probability above its prestimulus baseline are referred to as runs, whereas excursions below baseline are referred to as turns. SEM is shown by the gray bands. The dashed line between panels separates statistically significant monophasic responses from statistically significant biphasic responses (asterisks). Data were arcsine-transformed as described in Materials and Methods; this transformation accounts for uneven increments on the ordinates. Statistical significance (step vs no step) was assessed over the periods indicated by the horizontal lines above the traces; results for the short time window $(0-60 \mathrm{~s})$ are summarized and extended to other behavioral states in Table 1. Asterisks indicate significant differences ( $t$ test; $p<0.05$ ) between the means at each time point after correcting for multiple comparisons; plus symbols indicate significant comparisons detected in uncorrected $t$ tests. Late responses were considered biphasic only if significant in uncorrected $t$ tests. For completeness, the data from Figure 2 C are shown again in $\mathbf{G}$. There were at least 30 animals per treatment condition.

experiment. Second, the effect of concentration on steady-state behavior was smaller than the effect of concentration steps on transient responses. Although there was a significant main effect of concentration on steady-state behavior in the no-step control experiments $\left(F_{(5,175)}=4.32 ; p<10^{-4}\right)$, forward probability varied from 0.76 at $40 \mathrm{~mm} \mathrm{NaCl}$ to 0.84 at $150 \mathrm{~mm} \mathrm{NaCl}$, a range that is too small to account for the range of transient changes in forward probability. Hypothetically, the turn-and-run sequence should improve chemotaxis by directing the animal well away from the low-concentration region after an initial turn. Conversely, the sequence run-and-turn might impair chemotaxis by sending the animal back to the low-concentration region after the initial run, a possible explanation for the reduction in chemotaxis, shown at point $f$ in Figure 1. Below, we present the results of computer simulations that test the hypothetical effects of these two response sequences.

Step responses were nonlinear in three main ways. First, upstep run amplitude appeared to saturate with increasing step sizes. Second, as noted above, increases in step amplitude changed monophasic responses into biphasic ones. Third, step responses were not invertible, in that steps of equal amplitude but opposite sign did not produce mirror-image responses. The overall nonlinearity of step responses is significant, because it rules out classes of models based on linear systems theory and widely used in sensorimotor integration studies (Robinson, 1981; Bialek et al., 1991), including models of bacterial chemotaxis (Block et al., 1982). An alternative modeling strategy is presented below.

The early response components in the step-response assay (Fig. 3) were qualitatively consistent with the tendency of worms to accumulate in the highconcentration zone of the quadrant chemotaxis assay (Fig. 1). For example, a run triggered when a worm crosses a concentration border from low to high constitutes an approach response that would promote accumulation in the highconcentration zone. Similarly, a turn triggered when a worm crosses a border from high to low constitutes an avoidance response that tends to return the animal to the high-concentration zone. The state known as other was rare and did not appear to be modulated by concentration steps (Fig. $2 C$ and data not shown).

\section{Kinetic model of chemotaxis}

Although step responses were qualitatively consistent with chemotaxis in quadrant assays, the question remained as to whether step responses were quantitatively sufficient to account for this behavior. To address this issue, we developed an empirical model of step-response behavior.

The nondeterministic nature of locomotory transitions in $C$. elegans is evident from the raster plot of Figure $2 B(t=-100$ to $0 \mathrm{~s})$, which shows that worms switch between states with no evident correlations or periodicity. We therefore adopted a stochastic modeling approach in which it is assumed that transitions out of a state occur at a constant rate, regardless of how long the system has been in the state. We assessed this assumption, as shown in Figure 4A, by displaying the dwell-time distribution for each state as a log survivor plot (Haccou and Meelis, 1992). The plots were linear over most (96\%) of their range, indicating that the rate of leaving each behavioral state was approximately constant; we conclude that worm locomotion can be modeled stochastically, consistent with previous findings (Brockie et al., 2001). In the model, a worm was represented as a point that moved in a virtual Petri plate divided into quadrants, as shown in the behavioral assay in Figure 1. The 
Table 1. Statistical summary of the effects of concentration steps on the probability of the forward, reversal, and omega states

\begin{tabular}{|c|c|c|c|c|c|c|c|c|c|c|c|c|}
\hline \multirow{3}{*}{$\begin{array}{l}\text { Point in } \\
\text { Figure } 1\end{array}$} & \multirow{3}{*}{$\begin{array}{l}\text { Conc. } \\
\text { step }\end{array}$} & \multirow{3}{*}{$\begin{array}{l}\text { Baseline } \\
\text { conc. }\end{array}$} & \multirow{3}{*}{$\begin{array}{l}\text { Panel in } \\
\text { Figure } 3\end{array}$} & \multirow{2}{*}{\multicolumn{2}{|c|}{ Forward }} & \multicolumn{7}{|l|}{ Turns } \\
\hline & & & & & & \multicolumn{4}{|c|}{ Reversal } & \multicolumn{3}{|l|}{ Omega } \\
\hline & & & & $\Delta$ Probability $^{a}$ & ANOVA $^{b}$ & Signif. ${ }^{c}$ & $\Delta$ Probability & ANOVA $^{b}$ & Signif. $^{c}$ & $\Delta$ Probability $^{a}$ & ANOVA $^{b}$ & Signif. ${ }^{c}$ \\
\hline a & +50 & 0 & $E$ & + & $F_{(1,62)}=16.24$ & $* * *$ & - & $F_{(1,62)}=2.46$ & $* *$ & - & $F_{(1,62)}=8.48$ & ** \\
\hline $\mathrm{a}$ & -50 & 50 & $F$ & - & $F_{(1,108)}=9.75$ & $* * *$ & + & $F_{(1,108)}=13.93$ & $* * *$ & + & $F_{(1,108)}=6.19$ & $* *$ \\
\hline b & +10 & 40 & $A$ & + & $F_{(1,58)}=22.64$ & $* * *$ & - & $F_{(1,58)}=12.71$ & $* * *$ & - & $F_{(1,58)}=14.00$ & ** \\
\hline b & -10 & 50 & $B$ & $(-)$ & $F_{(1,68)}=3.78$ & ns & + & $F_{(1,68)}=5.16$ & $* * *$ & ns & $F_{(1,68)}=1.14$ & ns \\
\hline d & +10 & 50 & $C$ & + & $F_{(\underline{5,340)}}=2.33$ & * & ns & $F_{(1,68)}=0.11$ & ns & - & $F_{(\underline{5,340)})}=3.83$ & $* *$ \\
\hline$d$ & -10 & 60 & $D$ & - & $F_{(5,290)}=3.39$ & $* *$ & + & $F_{(5,290)}=3.34$ & ** & $(+)$ & $F_{(1,68)}=2.73$ & ns \\
\hline e & +50 & 50 & $G$ & + & $F_{(1,68)}=39.9$ & $* * *$ & - & $F_{(1,68)}=28.31$ & *** & - & $F_{(1,68)}=17.55$ & $* * *$ \\
\hline e & -50 & 100 & $H$ & - & $F_{(5,290)}=7.31$ & $* * *$ & + & $F_{(5,290)}=6.14$ & $* * *$ & $(+)$ & $F_{(1,58)}=2.084$ & ns \\
\hline$f$ & +100 & 50 & 1 & + & $F_{(1,68)}=28.32$ & $* * *$ & - & $F_{(1,68)}=18.78$ & *** & - & $F_{(1,68)}=14.87$ & $* * *$ \\
\hline$f$ & -100 & 150 & $J$ & - & $F_{(\underline{5,370)}}=2.46$ & $* * *$ & + & $F_{(\underline{5,370)}}=7.57$ & $* * *$ & - & $F_{(1,74)}=6.77$ & $* *$ \\
\hline
\end{tabular}

The table shows the results of a two-factor repeated-measures ANOVA comparing stimulated animals with unstimulated controls with regard to the early component of the responses (i.e., in the $60 \mathrm{~s}$ poststimulus time window indicated by the short horizontal lines in Fig. 3). There were at least 30 animals per treatment condition. The analysis shows that the forward state and the two turning states tended to be coordinately regulated.,$+ \Delta$ Probability $>0 ;-$, $\Delta$ probability $<0$; ns, ANOVA not significant; Conc., concentration. ${ }^{*} p<0.05$; ${ }^{* *} p<0.01$; ${ }^{* * *} p<0.001$.

${ }^{a}$ Parentheses indicate changes that were significant only in a post hoc $t$ test corrected for multiple comparisons.

${ }^{b}$ Underlining indicates that the $F$ value is for the treatment by time interaction; otherwise, the $F$ value is for the main effect of treatment.

Level of significance (Signif.) in the ANOVA.

speed of the model worm and the size of the virtual plate were scaled to match the conditions of the real quadrant assays. Behavioral state in the model was governed by the three-state Markovlike stochastic scheme shown in Figure $4 B$. The fourth behavioral state, other, was omitted from the model because it was rare and did not alter the worm's orientation. Given three states, there are six possible behavioral-state transitions. In the model, each type of transition caused a specific change in the direction of movement, as shown in Figure $4 C$, and as explained in Materials and Methods.

Rate constants were time and concentration dependent. For this reason, the model is not a Markov model in the strict sense. Other deviations from Markov behavior are discussed in Materials and Methods. The nature of the time and concentration dependence was determined empirically from the step-response experiments (Fig. 3) using the following relationship: $k_{i j}=f_{i j} / p_{i}$, where $k_{i j}$ is the rate for the transition from state $i$ to state $j, f_{i j}$ is the frequency of the $i$ to $j$ transition, and $p_{i}$ is the probability of finding a worm in state $i$. The quantity $k_{i j}$ was computed in consecutive $10 \mathrm{~s}$ bins to obtain its time course $k_{i j}(t)$. To facilitate the modeling, $k_{i j}(t)$ was expressed as the change in rate, $\Delta k_{i j}(t)$, relative to its prestep resting value. The bottom panels of Figure $4 D$ show the six time courses for $\Delta k_{i j}(t)$ in response to a $+50 \mathrm{~mm}$ step from a resting concentration of $50 \mathrm{~mm}$. Note that the main effect of the concentration step on the rate constants was to increase the rates of entry into the forward state with no effect on the exit rates, consistent with the observed increase in forward probability. Time courses of $\Delta k_{i j}(t)$ underlying each of the step responses shown in Figure 3 were obtained in the same way (data not shown). We tested the accuracy of $\Delta k_{i j}(t)$ computations in simulated step-response experiments (Fig. $4 D$, topmost panel) in which we recorded the behavioral state of 500 model worms in response to the $\Delta k_{i j}(t)$ waveforms shown in the bottom six panels in Figure $4 D$. The figure shows a good match between model and data; fits for the rate-constant functions underlying the other step responses were equally good (data not shown).

In simulating a quadrant assay, rate constants were initialized to their resting values and updated according to the history of border crossings as a simulated worm moved between quadrants on the plate. For example, when the worm moved from the lowconcentration to the high-concentration region in a virtual quadrant assay in which the home and test concentrations were 50 and
$100 \mathrm{~mm}$, respectively, we assumed that the rate constants changed according to the time courses shown in Figure $4 D$. However, border crossings were frequent on the time scale of the rateconstant changes; in most cases, the effect of one border crossing was not over before the next border crossing occurred. To accommodate frequent border crossings, we assumed that their effects combine by superposition in time [i.e., we convolved the $\Delta k_{i j}(t)$ functions with the history of border crossings]. A typical simulated worm track is shown in Figure $4 E$.

\section{Simulated quadrant assays}

We tested the model in simulated quadrant assays corresponding to points $\mathrm{a}-\mathrm{f}$ in the real quadrant assays of Figure 1. The model reproduced the two key features of the real assays: the inverse relationship between chemotaxis index and test concentration and the deviation from this trend at a test concentration of 150 $\mathrm{mm}$ (Fig. 5A). This result is significant because the model was adjusted to fit step responses (Fig. 3) and not the behavior of worms in spatial gradients. The fact that the model reproduced the deviation in the trend implies that the reduction in attraction to $150 \mathrm{~mm} \mathrm{NaCl}$ is attributable, at least in part, to differences in the step responses at this concentration; one possible difference is that the downstep turn lasts only $10 \mathrm{~s}$ at this concentration, as opposed to $\geq 20 \mathrm{~s}$ at the other concentrations. The overall correspondence between the model and the real data shows that it is possible to quantitatively reconstruct the behavior of worms in chemotaxis assays in terms of the kinetic parameters obtained from step responses of individual worms. We conclude that our kinetic analysis of step responses leads to an accurate and robust model of the sensorimotor transformation underlying $C$. elegans chemotaxis. We next used the model to probe the chemotaxis mechanism in ways that cannot currently be done in real worms.

\section{Virtual mutants}

The effects of upsteps and downsteps on locomotion are qualitatively consistent with attraction to higher salt concentrations, but it is unclear whether both types of responses are necessary for normal chemotaxis. To address this question, we created virtual mutants in which the response to upsteps or downsteps was specifically deleted in model worms, and we tested the virtual mutants in simulated quadrant assays. We found that deleting either the upstep or downstep response significantly reduced chemo- 
A

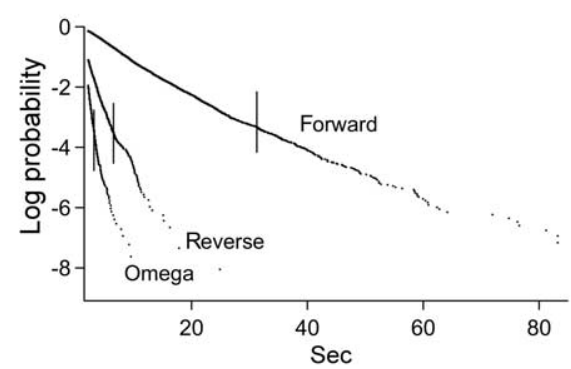

C
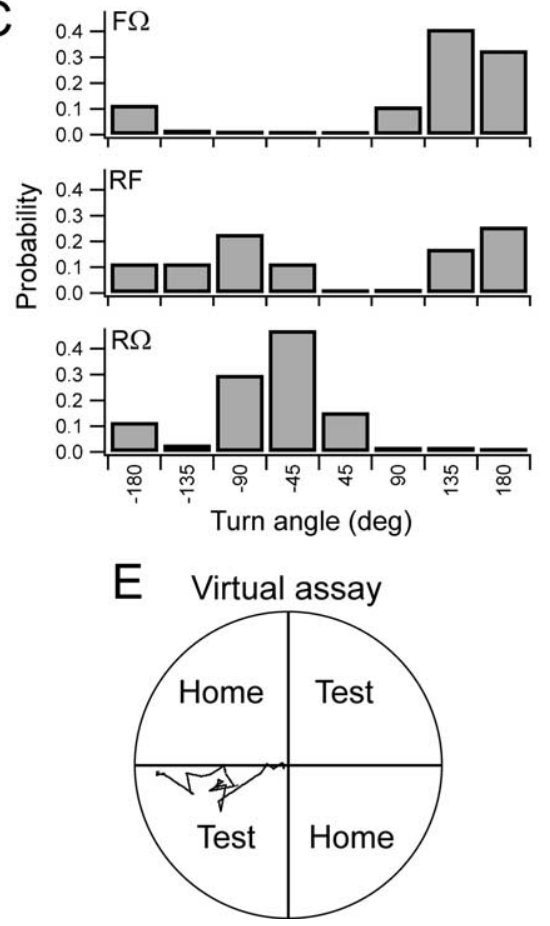

Figure 4. Kinetic model of chemotaxis. $\boldsymbol{A}$, Log survivor plots for forward, reversal, and omega states. These plots were constructed by computing the dwell-time distribution for each state and displaying on a natural log scale the probability of observing a dwell time more than or equal to to the duration indicated on the abscissa. Many points overlap on this scale; hatch marks indicate the point to the left of which $96 \%$ of the data occur. Dwell times $\leq 2$ s were omitted from this analysis because of limitations on observer reaction time. Data were pooled from all of the no-step control experiments in Figure 3 . The overall linearity of the data indicates a constant exit rate for each state. $\boldsymbol{B}$, Behavioral-state diagram with rate constants $(k)$. $F$, Forward; $\mathrm{R}$, reversal; $\Omega$, omega turn. $C$, Probability density functions for turn angles associated with the $F \Omega$, RF, and $R \Omega$ behavioral-state transitions. These functions were determined empirically by measuring by eye, in video playback, the direction of motion before and after turns. The other three transitions were modeled in idealized form, as described in Materials and Methods. deg, Degrees. D, Forward probability and changes in rate constants. The topmost panel shows a comparison of computed (model) and actual (data) forward probability for a simulated step-response experiment in which the rate-constant time courses were played back through the kinetic model. The model trace represents average forward probability for 500 step responses. The other panels show the rate constants as a function of time relative to the concentration step indicated by the arrowhead. Data are from Figure $3 G$. $\boldsymbol{E}$, Diagram of the virtual quadrant assay showing the track of a simulated worm run for the equivalent of 10 min. Home concentration, $50 \mathrm{~mm} \mathrm{NaCl}$; test concentration, $100 \mathrm{~mm} \mathrm{NaCl}$.

taxis efficiency (Fig. 5B) (WT model vs downstep response deleted, $F_{(1,35)}=184.90, p<0.001 ;$ WT model vs upstep response deleted, $\left.F_{(1,35)}=382.06, p<0.001\right)$. We conclude that both upstep and downstep responses make quantitatively detectable contributions to chemotaxis.

We also used the virtual-mutant approach to probe the relative contributions to chemotaxis of the late components in the biphasic sequences identified in Figure $3 F, H-J$. As noted above, the response to large downsteps is the sequence turn-and-run. Does the run in this sequence improve chemotaxis? To answer this question, we specifically deleted the run from the turn-andrun sequence. This was done by setting $\Delta k_{i j}(t)$ waveforms to zero immediately after the turn. Testing such mutants in simulated quadrant assays (Fig. 5C), we found that deleting the run from the turn-and-run sequence caused a statistically detectable but small change in chemotaxis at test concentrations of $0 \mathrm{mM}\left(t_{(4)}=27.24 ; p<10^{-4}\right)$ and $100 \mathrm{~mm}\left(t_{(4)}=8.36 ; p<0.002\right)$ and no effect at $150 \mathrm{mM}\left(t_{(4)}=2.71 ; p>0.05\right)$. Because the changes were so small, it is unlikely that the second phase of the turnand-run sequence makes a substantial contribution to the effectiveness of downstep responses in quadrant assays.

We used a similar approach to address the question of whether the turn in the run-and-turn sequence, which appears in the response to the largest upstep (Fig. 3I), impairs chemotaxis. Deleting the turn from this sequence had no effect on chemotaxis (data not shown; $I=0.31 \pm 0.015$ vs $\left.0.32 \pm 0.007 ; t_{(4)}=0.53 ; p>0.05\right)$. Thus, it is unlikely that postrun turns contribute to the impairment of chemotaxis. Together, the absence of salient effects of deleting the late components of biphasic responses suggests that chemotaxis is mainly the result of the early response components, with little or no contribution from the late components.

\section{Step responses are \\ experience dependent}

The comprehensive step-response data of Figure 3 provide a standard of comparison for determining the effects of various treatments that probe the neuronal basis of the sensorimotor transformation. Previous studies have shown that preexposure to various $\mathrm{Na}^{+}$and $\mathrm{Cl}^{-}$salts causes adaptation, measured as a reduction in chemotaxis to these ions in radial gradients (Jansen et al., 2002). Because adaptation is often viewed as the attenuation of sensory responses, one might expect that preexposure would merely reduce the overall amplitude of the step-response waveform. To test this hypothesis, we compared the $\pm 100 \mathrm{~mm}$ step responses of worms preexposed to a high concentration of $\mathrm{NaCl}$ (150 $\mathrm{mm}$ for $10 \mathrm{~min}$ ) against the step responses of control worms preexposed to the home concentration (50 $\mathrm{mm}$ for $10 \mathrm{~min}$ ). Contrary to expectations, preexposure to $150 \mathrm{~mm} \mathrm{NaCl}$ specifically eliminated the early phases of the upstep and downstep responses, leaving the later phases intact (Fig. $6 \mathrm{~A}$ ). This result is consistent with a model in which the early and late phases of the step responses are mediated by distinct neuronal pathways with different sensitivities to adaptation.

After preexposure to high salt, the overall effect of an upstep became a turn, and the overall effect of a downstep became a run. This pattern of response is consistent in principle with repulsion from regions of high concentration. To test whether the altered step responses were quantitatively sufficient to produce repulsion, we extracted the time courses $\Delta k_{i j}(t)$ from the $150 \mathrm{~mm}$ preexposure 
condition and used them in simulated quadrant assays (test concentration, $150 \mathrm{~mm}$ ). Preexposure resulted in a chemotaxis index near zero $(I=0.0052 \pm 0.016$; $n=3$ simulated assays), indicating that, although preexposure does not transform the net effect of step responses from accumulation to repulsion, it is sufficient to eliminate chemotaxis.

\section{Neuronal basis of step responses}

Neuronal ablation provides a second means of probing the function of neuronal pathways. Previous studies have shown that ablation of the ASE chemosensory neurons reduces chemotaxis to soluble attractants in radial gradients to a greater extent than ablation of any other chemosensory neurons in the animal (Bargmann and Horvitz, 1991). Nevertheless, little is known about how ASE functions to promote chemotaxis. To address this issue, we measured step responses of worms in which the contribution of ASE neurons was removed in two different ways: by laser ablation or by a mutation in the che-1 gene, a transcription factor required for specification of the ASE neurons (Chang et al., 2003; Uchida et al., 2003).

Surprisingly, ablation of ASE specifically deleted a singleresponse component (Fig. 6B). Comparison of step responses in ablated animals and sham-operated controls showed that the downstep turn was eliminated, whereas the upstep run was unaffected. In contrast, comparison of ablated animals and no-step controls showed that the late phase of the downstep response (i.e., the run) was still present. Thus, only the downstep turn was affected by the ablation.

A qualitatively similar pattern of results was observed in che-1 mutants (Fig. 6C). Comparison of step responses in mutants and wild-type animals showed that the downstep turn was eliminated. In contrast, comparison of mutants and no-step mutant controls showed that the upstep run remained, as did the late phase of the downstep response. The only difference between the effects of the ASE ablation and the che-1 mutation was that the latter caused attenuation of the upstep run (Fig. 6C1). This difference could be attributable to the fact that che-1 is expressed in several neurons in addition to ASE (Uchida et al., 2003). Together, these results suggest that ASE activity is the major determinant of the downstep turn but not of the other response components.

\section{Discussion}

The main goal of this study was to investigate the step response of the C. elegans chemotaxis mechanism. To measure step responses in C. elegans, we devised a concentration clamp that delivers rapid changes in attractant concentration to unrestrained worms crawling on a typical substrate. Using this new device, we found that the step response is consistent with accumulation in regions of high attractant concentration, exhibits distinct early and late phases, varies nonlinearly with the sign and amplitude of the step, and is altered by experience and neuronal ablation. These results substantiate the pirouette model of C. elegans chemotaxis (PierceShimomura et al., 1999) by demonstrating directly that sudden changes in concentration regulate turning behavior. In addition, these results offer some of the first insights into how the transfer function might be computed at the level of individual neurons.

The present study builds on a previous one that examined step responses in swimming worms (Dusenbery, 1980). In the previ-
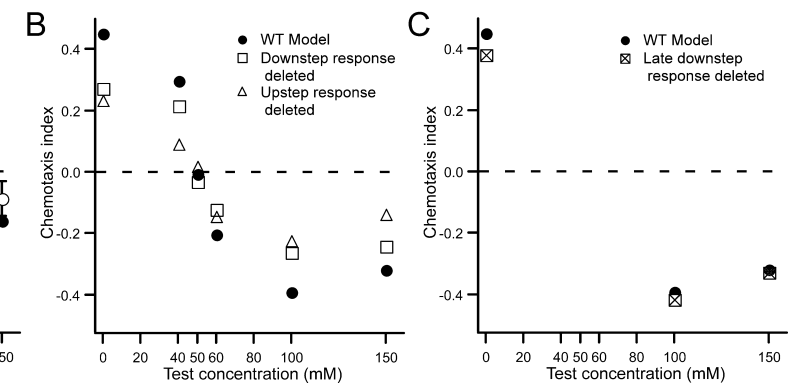

Average chemotaxis index versus test concentration in simulated quadrant assays. Data points for simulated assays represent the mean of three assays of 2000 worms each; SE was typically less than or equal to symbol height. Symbols are given in each key. $\boldsymbol{A}$, Wild-type model versus data from the real quadrant assays. There are no model data points at test concentrations which the turn-and-run sequence was observed (Fig. $3 F, H, J)$.

ous study, worms were restrained by the tail and submerged in a pool of saline. Under these conditions, worms swim rather than crawl, but correlates of forward and reverse body waves, like those that propel a crawling worm, are still detectable. Rapid decreases in salt concentration $(-25$ or $-50 \mathrm{~mm})$ produced an increase in the probability of reverse body waves, whereas rapid increases $(+25$ or $+50 \mathrm{~mm}$ ) had the opposite effect. The present study measured step responses in unrestrained crawling worms and investigated a larger region of the input space ( \pm 10 to \pm 100 $\mathrm{mm}$ ). Under these conditions, the step responses were found to extend to an additional locomotory state (omega turns) and had time courses that were more complex. Nevertheless, the swimming and crawling sensorimotor transformations share the overall pattern of avoidance responses when the concentration drops and approach responses when the concentration rises. Thus, chemosensory input appears to regulate locomotory behavior in swimming and crawling worms in similar ways.

The concentration-clamp approach has also been applied to bacterial chemotaxis (Block et al., 1982; Segall et al., 1986). In bacteria, an impulse elicits a transient change in the probability of tumbling, a form of turning behavior analogous to pirouettes in C. elegans. In contrast to the results presented here, the sensorimotor transformation in bacteria is linear: the impulse response is invertible and scales linearly with stimulus amplitude, and the step response can be predicted by convolving the impulse response with a step input. A simple explanation for the difference in linearity is that the stimuli used in the bacteria work were small, producing responses well below saturation, whereas the stimuli used in the present work produced responses at or near the saturation point. Alternatively, the difference in linearity may reflect fundamental differences in sensorimotor integration in the two systems.

\section{Quantitative reconstruction of behavior}

The chemotaxis sensorimotor transformation can be described in terms of a simple three-state stochastic model. Transitions among the three states are governed by first-order rate constants that respond transiently to step changes in attractant concentration. The linearity of the bacterial sensorimotor transformation made it possible to compute the time course of state probability simply by convolving sensory input with the impulse response expressed in terms of the time course of state probability. In contrast, the nonlinearity of the $C$. elegans transformation required us to adopt a two-stage procedure in which sensory input 


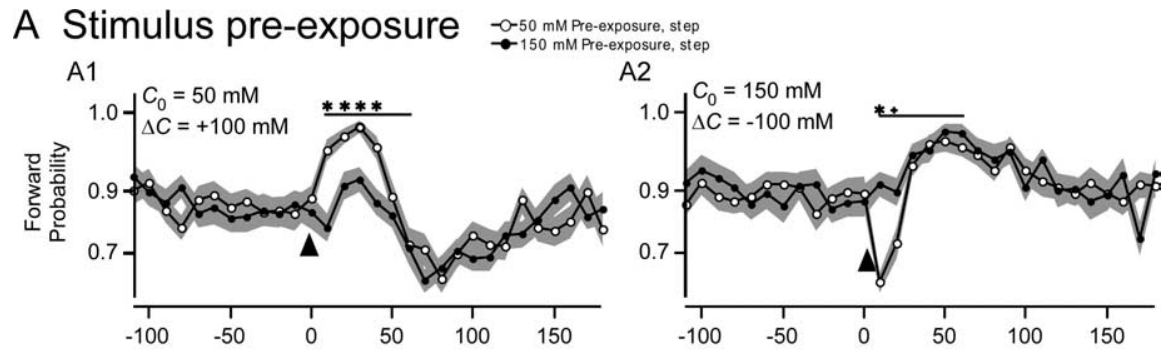

B ASE ablation $\underset{\substack{-0-\text { Sham, step } \\ \rightarrow-A S E ; \text { step }}}{\rightarrow-S}$
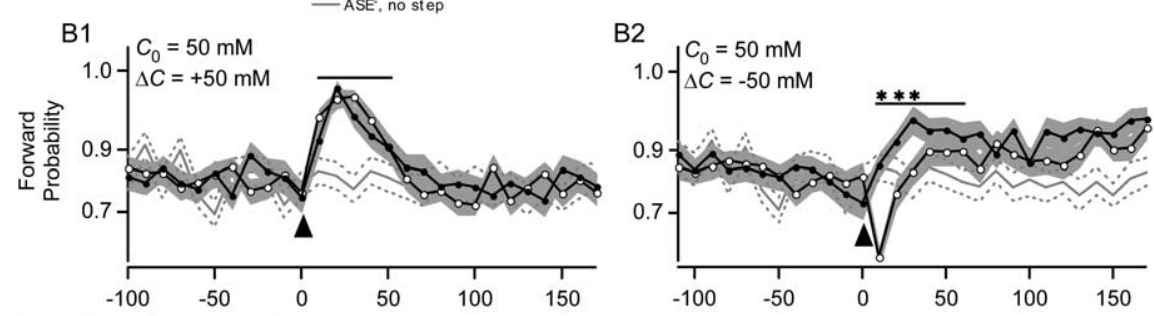

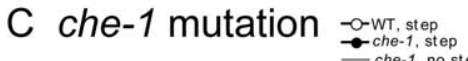
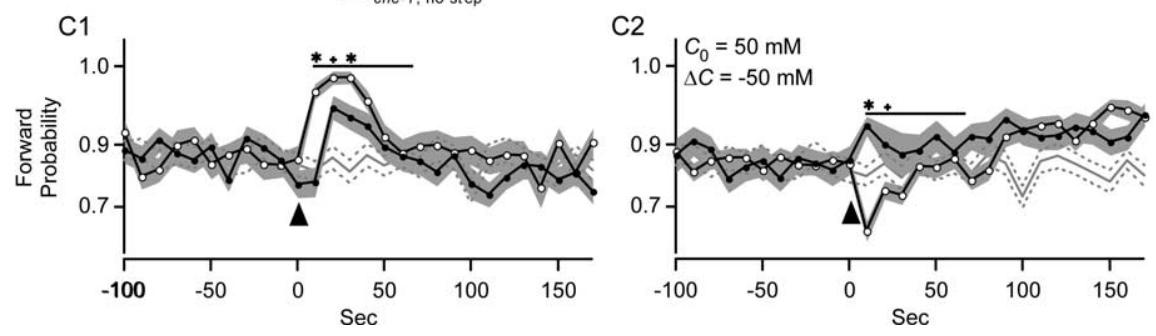

Figure 6. Neuronal basis of step responses. Each panel shows the time course of average forward-state probability as a function of time for the baseline $\mathrm{NaCl}$ concentration $\left(C_{0}\right)$ and concentration step $(\Delta C)$ shown in the top left corner. Symbols are given in the keys. Arrowheads indicate the time of the concentration step. In control experiments (no step), both showerheads emitted the baseline concentration. SEM is shown by the gray bands or by dashed gray lines. $\boldsymbol{A}$, Stimulus preexposure. Preexposing worms to $150 \mathrm{~mm} \mathrm{NaCl}$ specifically eliminated the early phase of the response to upsteps $\left(\boldsymbol{A 1}, \boldsymbol{F}_{(1,58)}=20.2 ; p<10^{-4}\right.$ and asterisks) and to downsteps $\left(\boldsymbol{A} 2, F_{(1,58)}=12.9 ; p<10^{-3}\right.$ and asterisks). $\boldsymbol{B}$, ASE ablation. Removing ASE neurons had no effect on upstep responses $\left(\boldsymbol{B} 1, \mathrm{ASE}^{-}\right.$, step vs sham, step; $\left.F_{(1,67)}=0.16 ; p>0.05\right)$ but completely eliminated the downstep turn $(\boldsymbol{B} 2$, ASE, step vs sham, step; $F_{(1,64)}=16.0 ; p<10^{-3}$ and asterisks). In contrast, the downstep run was still present [B2, ASE ${ }^{-}$, step vs ASE ${ }^{-}$, no step; $0-170$ s poststimulus (poststim); $\left.F_{(1,62)}=51.84 ; p<10^{-8}\right]$, as was the upstep run $\left(\boldsymbol{B}_{1}\right.$, ASE $^{-}$, step vs ASE ${ }^{-}$, no step; $0-60$ s poststim; $\left.F_{(1,65)}=28.35 ; p<10^{-5}\right)$. $\boldsymbol{C}$, The che-1 mutation. The mutation eliminated the downstep turn $(\boldsymbol{C}$, che-1, step vs WT, step; $F_{(1,98)}=12.2 ; p<10^{-3}$ and asterisks). In contrast, the downstep run was still present ( $\mathbf{C}$, che-1, step vs che-1, no step; $0-170$ s poststim; $\left.F_{(1,70)}=21.17 ; p<10^{-4}\right)$, as was the upstep run ( $\mathbf{C}$, che-1, step vs che-1, no step; $0-60$ s poststim; $\left.F_{(1,70)}=7.8 ; p<0.01\right)$. The mutation did, however, attenuate the upstep run $\left(\boldsymbol{C} \mathbf{1}\right.$, che-1, step vs WT, step; $F_{(1,58)}=$ 15.0; $p<10^{-3}$ and asterisks). Data were arcsine-transformed, as described in Materials and Methods; this transformation accounts for uneven increments on the ordinates. Statistical significance was assessed over the periods indicated by the horizontal lines above the traces. Asterisks indicate significant differences ( $t$ test; $p<0.05$ ) between the means at each time point after correcting for multiple comparisons; + symbols indicate significant comparisons detected in uncorrected $t$ tests. There were at least 30 animals per treatment condition.

is convolved with the step response of the rate constants and the rate constants are updated to compute state probabilities. The model was fully constrained (contained no free parameters) and designed to predict the step responses of individual worms. Nevertheless, it accurately reproduced the absolute levels of chemotaxis in the quadrant assay, including the observed relationship between test concentration and chemotaxis efficiency, as well as the deviation from this relationship at high test concentrations. We conclude that step responses are quantitatively sufficient for chemotaxis in steep gradients.

The success of the model enabled us to assign relative contributions to chemotaxis of different components of the step responses by specifically deleting particular components from the model in the so-called virtual-mutant approach. This approach yielded two main findings. First, we found that responses to both increases and decreases in concentration are essential for normal levels of chemotaxis. Second, we found that chemotaxis depends primarily on the early phase of the response (i.e., the turn elicited by downsteps and the run elicited by upsteps). A turn produces large changes in orientation (Fig. 4C) (Croll, 1976; Pierce-Shimomura et al., 1999; Hills et al., 2004) and so constitutes an avoidance response, whereas a run suppresses changes in orientation and so constitutes and approach response. Thus, chemotaxis in steep gradients appears to be the product of approach and avoidance behaviors, as inferred previously from statistical analyses of worms crawling in radial gradients (Pierce-Shimomura et al., 1999).

\section{Chemotaxis in radial gradients}

The instantaneous rate of concentration change in the present study is much larger than the rate of change in shallow lowamplitude gradients, such as those of the frequently used radial gradient assay (Ward, 1973). In our experiments, the minimum step amplitude (10 mM) corresponds to a rate of $\sim 10 \mathrm{~mm} / \mathrm{s}$, whereas in a radial gradient assay, the worm experiences rates of $\sim 10^{-2} \mathrm{~mm} / \mathrm{s}$ or less (PierceShimomura et al., 1999). This raises the interesting question of what the step response looks like in the case of lowamplitude stimuli and whether simply convolving step responses with stimulus history can explain chemotaxis in a radial gradient as well as it does in a quadrant assay. This issue could be addressed by stimulating worms with steps smaller than the ones used here, but improvements in the signal-to-noise ratio of the concentration clamp will be necessary before this experiment becomes practical. Nevertheless, two treatments that we found to reduce step responses have been shown previously to reduce chemotaxis in radial gradients. Ablation of ASE neurons, or mutation of the che-1 gene, eliminates the early component of the downstep response (Fig. $6 B, C)$, and previous work has shown that identical treatments reduce the level of chemotaxis in radial gradients (Dusenbery et al., 1975; Bargmann and Horvitz, 1991; Pierce-Shimomura et al., 2001; Chang et al., 2004). This correlation is consistent with the hypothesis that step-response behavior reflects a single unified chemotaxis mechanism.

\section{Neuronal mechanisms}

Our observation that stimulus preexposure eliminates the early phase of step responses but leaves the later phase intact implies parallel pathways for chemosensory processing (Fig. 6A). The early phase of the step response was apparent at all stimulus amplitudes, whereas the late phase appeared only at higher stimulus amplitudes (Fig. 3) ( $+100 \mathrm{~mm}$ for upsteps and -50 or $-100 \mathrm{~mm}$ for downsteps). We therefore propose a model in which chemo- 
sensory processing involves a low-threshold pathway that regulates the early response and desensitizes during stimulus preexposure and a high-threshold pathway that regulates the late response and resists desensitization. The function of the lowthreshold pathway is to promote chemotaxis, because it generates runs in response to upsteps and turns in response to downsteps. Given that the high-threshold pathway generates the opposite pattern of responses (turns in response to upsteps and runs in response to downsteps), it would appear to drive repulsion, and prolonged exposure to $\mathrm{NaCl}$ has been shown to transform this cue into a repellent (Jansen et al., 2002). However, quadrant assay simulations using step responses from the preexposure experiment indicated that the high-threshold responses are not sufficient for repulsion. The function of the high-threshold pathway therefore remains a question for additional study.

The low-threshold pathway may itself be divisible into distinct pathways for upsteps and downsteps. This follows from the fact that removing the function of the ASE chemosensory neurons, whether by ablation or genetic manipulation, specifically deletes the downstep turns, leaving the upstep runs relatively intact. We therefore propose a model in which chemotaxis is the joint product of approach and avoidance behaviors (supplemental Fig. 1, available at www.jneurosci.org as supplemental material). The ASE neurons detect concentration decreases and elevate the probability of turning states to produce avoidance responses. Other chemosensory neurons detect concentration increases and elevate the probability of runs to produce approach responses. Based on previous laser-ablation studies in radial gradient assays (Bargmann and Horvitz, 1991), the chemosensory neurons ADF, ASI, and ASG are the most likely candidates for the chemosensory neurons of the upstep pathway. This is because the joint ablation of these neurons is required to eliminate all residual $\mathrm{Na}^{+}$or $\mathrm{Cl}^{-}$ chemotaxis ability after an ASE ablation. Previous work has shown that the left and right ASE neurons (ASEL and ASER), although anatomically homologous, are genetically and functionally distinct: ASEL is specialized for the detection of $\mathrm{Na}^{+}$ ions, whereas ASER is specialized for the detection of $\mathrm{Cl}^{-}$ions (Yu et al., 1997; Pierce-Shimomura et al., 2001). This finding raises the question of whether ASEL and ASER respond similarly to upsteps and downsteps in $\mathrm{NaCl}$ concentration. Unilateral ablations of ASE neurons can now be used to address this issue.

The concentration clamp emerges as a useful tool in the neuronal analysis of chemosensory behavior in C. elegans. Its key strength lies in the ability to resolve distinct components in what had been seen previously as a single phenomenon. These components include the different responses to concentration increases and decreases, as well as the early and late phases of these responses. Combined with neuronal ablations and genetic mutations, the assay allowed us to assign specific functions to identified neurons with a role in the behavior that was less well defined previously. By studying the effects on the step response of ablating other sensory neurons, as well as downstream interneurons and motor neurons, it should be possible to identify additional neurons in the chemotaxis network and to assign specific functions to them.

\section{References}

Bargmann CI, Avery L (1995) Laser killing of cells in Caenorhabditis elegans. In: Methods in cell biology (Epstein HF, Shakes DC, eds), pp 225-250. San Diego: Academic.

Bargmann CI, Horvitz HR (1991) Chemosensory neurons with overlapping functions direct chemotaxis to multiple chemicals in C. elegans. Neuron 7:729-742.
Bialek W, Rieke F, de Ruyter van Steveninck RR, Warland D (1991) Reading a neural code. Science 252:1854-1857.

Block SM, Segall JE, Berg HC (1982) Impulse responses in bacterial chemotaxis. Cell 31:215-226.

Brenner S (1974) The genetics of Caenorhabditis elegans. Genetics 77:71-94. Brockie PJ, Mellem JE, Hills T, Madsen DM, Maricq AV (2001) The C. elegans glutamate receptor subunit NMR-1 is required for slow NMDAactivated currents that regulate reversal frequency during locomotion. Neuron 31:617-630.

Chang S, Johnston Jr RJ, Hobert O (2003) A transcriptional regulatory cascade that controls left/right asymmetry in chemosensory neurons of $C$. elegans. Genes Dev 17:2123-2137.

Chang S, Johnston Jr RJ, Frokjaer-Jensen C, Lockery S, Hobert O (2004) MicroRNAs act sequentially and asymmetrically to control chemosensory laterality in the nematode. Nature 430:785-789.

Colquhoun D, Hawkes AG (1995) A Q-matrix cookbook. In: Single-channel recording, Ed 2 (Sakmann B, Neher E, eds), pp 589-633. New York: Plenum.

Croll NA (1975) Components and patterns in the behavior of the nematode Caenorhabditis elegans. J Zool 176:159-176.

Croll NA (1976) When C. elegans (nematoda: rhabditidae) bumps into a bead. Can J Zool 54:566-570.

Dusenbery DB (1980) Responses of the nematode Caenorhabditis elegans to controlled chemical stimulation. J Comp Physiol 136:127-331.

Dusenbery DB, Sheridan RE, Russell RL (1975) Chemotaxis-defective mutants of the nematode Caenorhabditis elegans. Genetics 80:297-309.

Haccou P, Meelis E (1992) Statistical analysis of behavioral data. Oxford: Oxford UP.

Hedgecock EM, Russell RL (1975) Normal and mutant thermotaxis in the nematode Caenorhabditis elegans. Proc Natl Acad Sci USA 72:4061-4065.

Hills T, Brockie PJ, Maricq AV (2004) Dopamine and glutamate control area-restricted search behavior in Caenorhabditis elegans. J Neurosci 24:1217-1225.

Jansen G, Weinkove D, Plasterk RH (2002) The G-protein gamma subunit gpc-1 of the nematode C. elegans is involved in taste adaptation. EMBO J 21:986-994.

Pierce-Shimomura JT, Morse TM, Lockery SR (1999) The fundamental role of pirouettes in Caenorhabditis elegans chemotaxis. J Neurosci 19:9557-9569.

Pierce-Shimomura JT, Faumont S, Gaston MR, Pearson BJ, Lockery SR (2001) The homeobox gene lim-6 is required for distinct chemosensory representations in C. elegans. Nature 410:694-698.

Robinson DA (1981) The use of control systems analysis in the neurophysiology of eye movements. Annu Rev Neurosci 4:463-503.

Rutherford TA, Croll NA (1979) Wave forms of C. elegans in a chemical attractant and repellent and in thermal gradients. J Nematol 11:232-240.

Segall JE, Block SM, Berg HC (1986) Temporal comparisons in bacterial chemotaxis. Proc Natl Acad Sci USA 83:8987-8991.

Uchida O, Nakano H, Koga M, Ohshima Y (2003) The C. elegans che-1 gene encodes a zinc finger transcription factor required for specification of the ASE chemosensory neurons. Development 130:1215-1224.

Ward S (1973) Chemotaxis by the nematode Caenorhabditis elegans: identification of attractants and analysis of the response by use of mutants. Proc Natl Acad Sci USA 70:817-821.

Ward S, Thomson N, White JG, Brenner S (1975) Electron microscopical reconstruction of the anterior sensory anatomy of the nematode Caenorhabditis elegans. J Comp Neurol 160:313-338.

Ware RW, Clark D, Crossland K, Russell RL (1975) The nerve ring of the nematode Caenorhabditis elegans: sensory input and motor output. J Comp Neurol 162:71-100.

Wicks SR, de Vries CJ, van Luenen HG, Plasterk RH (2000) CHE-3, a cytosolic dynein heavy chain, is required for sensory cilia structure and function in Caenorhabditis elegans. Dev Biol 221:295-307.

Winer BJ, Brown DR, Michels KM (1991) Statistical principles in experimental design, Ed 3, p 356. Boston: McGraw-Hill.

Yu S, Avery L, Baude E, Garbers DL (1997) Guanylyl cyclase expression in specific sensory neurons: a new family of chemosensory receptors. Proc Natl Acad Sci USA 94:3384-3387.

Zhao B, Khare P, Feldman L, Dent JA (2003) Reversal frequency in Caenorhabditis elegans represents an integrated response to the state of the animal and its environment. J Neurosci 23:5319-5328. 